\title{
The method for optimisation of gas compressors performance in gas storage systems
}

\author{
Michał Warchoł \\ Transition Technologies S.A., \\ Warsaw, Poland \\ Email: m.warchol@tt.com.pl
}

\section{Konrad Świrski}

Institute of Heat Engineering,

Warsaw University of Technology, Poland

Email: konrad.swirski@itc.pw.edu.pl

\section{Błazej Ruszczycki}

Nencki Institute of Experimental Biology, Polish Academy of Sciences,

Warsaw, Poland

Email: bruszczy@nencki.gov.pl

\section{Konrad Wojdan*}

Institute of Heat Engineering, Warsaw University of Technology, Poland

Email: konrad.wojdan@itc.pw.edu.pl

*Corresponding author

\begin{abstract}
We present a computational method for achieving an optimal operation of compressor units used in industrial gas storage systems. The proposed method is capable to operate with a mix of compressor types (i.e., with different operational parameters, different power drives, and different types of construction, e.g., reciprocal and turbocompressors). The goal of the optimisation is to find an optimal compressors configuration and distribution of the compressor loads for each instance of time. The proposed method is based on conversion of a multidimensional discrete-continuous optimisation problem into a set of independent combinatorial and nonlinear optimisation problems. We derive the mathematical foundations of the algorithms. The exemplary results of the application are presented. [Received: May 4, 2016; Accepted: December 2, 2016]
\end{abstract}

Keywords: optimisation; compressor station; dynamic programming; sequential quadratic programming; SQP; gas storage.

Reference to this paper should be made as follows: Warchoł, M., Świrski, K., Ruszczycki, B. and Wojdan, K. (2018) 'The method for optimisation of gas compressors performance in gas storage systems', Int. J. Oil, Gas and Coal Technology, Vol. 17, No. 1, pp.12-33. 
Biographical notes: Michał Warchoł received his $\mathrm{PhD}$ in 2003 in Automation and Robotics, at the Warsaw University of Technology. He currently works as a Scientific Consultant in Transition Technologies S.A. His areas of interest include advanced control, optimisation algorithms and thermodynamic calculations.

Konrad Świrsk is a Professor at Warsaw University of Technology, Institute of Heat Engineering. He received his $\mathrm{PhD}$ in 1995 and become a Professor in 2010. His research and implementation topics are focused on energy market, optimisation and modelling of heat processes, IT systems in power and gas industry.

Błazej Ruszczycki obtained his PhD degree in 2007 from the University of Miami, Physics Department. Subsequently, he joined the group led by Neil F. Johnson, working on various aspects of complex systems, with an emphasis on the modelling of clustering phenomena. His current interests in computational techniques include image recognition and analysis techniques, optimisation of experimental setups and agent-based modelling. He currently focuses on biological imaging techniques working at the Nencki Institute of Experimental Biology.

Konrad Wojdan is an Assistant Professor at Warsaw University of Technology, Institute of Heat Engineering. He received his PhD in 2009 in Automation and Robotics at the Warsaw University of Technology. His research and implementation topics are focused on advanced control, optimisation and modelling of industrial processes as well as applications of machine learning methods to power and gas industry.

\section{Introduction}

Dynamically changing gas market demands and the growing necessity to secure strategic gas reserves, elevates the importance of gas storage plants in modern natural gas systems. The compressor units are the key devices in the gas storage plant. Their operational limits dictate the plant's withdrawal and injection capabilities. When the gas pressure difference between the pipeline and the reservoir (e.g., gas caverns) is large, the compressors are the bottleneck limiting the gas flow through the storage plant, Wojdan et al. (2014). The proper determination of the compressor flow limit is therefore essential to run the plant effectively. The cost of energy consumed to run the compressors is a significant contribution to the operational costs, see Kurz et al. (2012). For a complex gas storage plant, with multiple compressors in operation, the load distribution between compressors impacts the plant total energy consumption. Thus, the goal of the compressor performance optimisation is to find an optimal configuration and distribution of the load on each compressor for each instance of time so that operational cost is minimised under the constraints of contractual obligations.

The main problem in finding an optimal configuration of the compressor station is that we encounter both discrete and continuous variables, subject to a set of operational and technical constraints, mostly determined by the surge and the choke lines. Several different numerical approaches were applied to find a solution of such a complex optimisation problem. Osiadacz (1980) and Carter (1996) propose to apply mixed-integer-nonlinear programming method in order to minimise the fuel consumption 
of gas driven compressors. Rose at al. (2016) study theoretically discrete-continuous models (especially mixed-integer and general disjunctive programming models) for the problem of minimum cost compressor station operation. Wong and Larson (1968) minimise the total fuel cost incurred by compressor stations via dynamic programming. The application of a genetic algorithm for optimisation of compressor gas performance is presented by Habibvand and Behbahan (2012), Elshiekh (2014) and Hawryluk et al. (2010). Zhang and $\mathrm{Wu}$ (2015) discusses data from minimisation of compressor energy cost by means of modified genetic algorithms. Xu et al. (2012) propose a model based on bundle projection sub-gradient method combined with Lagrange multiplier. Applicability and advantages of different compressors drives is discussed by Almasi (2011). The performance maps for centrifugal compressor are investigated in detail by Cortinovis et al. (2014). More reviews can be found in Rios-Mercadoa nd Borraz-Sanchez (2015) and Koch et al. (2015).

Figure 1 Two sections of the axial turbocompressor, driven by the same shaft (e.g., by the same electric motor or gas turbine), can be connected in parallel (valve b closed) or in serial mode (valves a and c closed) (see online version for colours)

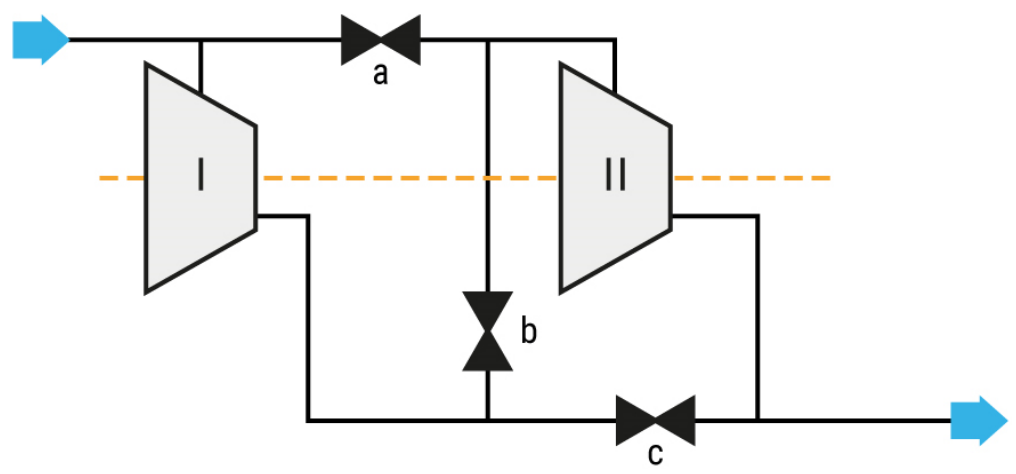

Figure 2 Compressor block comprising two axial compressors (one driven by electric motor, and one driven by gas turbine) and two reciprocating compressors (driven by gas turbines) (see online version for colours)

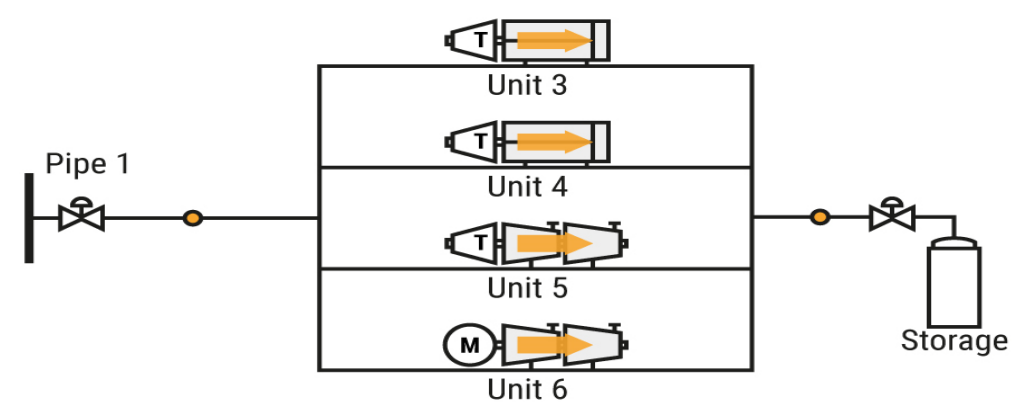

In this article we propose a novel approach based on conversion of a complex multidimensional discrete-continuous optimisation problem into a set of independent combinatorial nonlinear optimisation problems that are solved by dynamic programming, and sequential quadratic programming (SQP), respectively. We present an optimisation 
method that operates with commonly used combinations of compressors and compressor drives, including electric- and gas-driven turbocompressors (centrifugal, axial or mixed) and reciprocating compressors. The method also supports serial and parallel connection of compressor sections, see Figure 1, and operation in bypass mode. The objective of the article is to break the optimisation problem into a set of individual, less complex sub-problems. Therefore, we need to analyse separately different cases, e.g., reciprocating and turbocompressors in parallel connection. This comes at the price of a longer analytical formalism, yet allows us to apply faster numerical procedures. We concentrate primarily on $N$ compressors working in parallel (see Figure 2) which is the basic topology of connections between the compressors in gas storage units. The presented approach may be generalised to more complex topologies, we outline the generalisation to the cascade connection of compressors. For the reason of simplicity, we discus only the case when the gas plant is connected to one external pipeline. The case of more than one pipeline can be handled by treating it as a multiple independent optimisation problem.

In the following article we focus on the model of the compressor block which contains all compressors in the plant together with engines powering the compressors. In the model of the compressor block we include the gas coolers while other above ground devices present in the gas plant, such as glycol absorption columns used in the withdrawal phase are considered separately. The conditions in which the compressor block operates are described mainly by the suction pressure $p^{s}$, discharge pressure $p^{d}$, the gas suction temperature and the gas composition, further details are given in Section 2.

Industrial gas storages operate with large ranges of gas flowand large ranges of reservoir pipeline pressure difference. Therefore, the combination of reciprocating and turbocompressors (e.g., see Figure 2), in different configurations, is commonly used to secure the effective operation in the entire range of operational conditions, e.g., see Woelfe and Leuning (2004).

The most common types of compressors used in the industrial gas storages are:

- reciprocating compressors, used in the case of low gas flow when the required pressure ratio is high. The maximal power is up to $30 \mathrm{MW}$, the pressure ratio per compressor stage is 4 , the shaft rotational speed $500-1,800 \mathrm{rev} / \mathrm{min}$

- multistage axial turbocompressors (up to 15 stages), typically used in the case of large flows $\left(>200,000 \mathrm{~m}^{3} / \mathrm{h}\right)$ and low pressure ratio (1.5 per stage), rotational speed $5,000-8000 \mathrm{rev} / \mathrm{min}$

- multistage centrifugal turbocompressors (three to ten stages), used in the largest plants (flows $>500,000 \mathrm{~m}^{3} / \mathrm{h}$ ), pressure ratio per stage 2 , rotational speed $6,000-20,000 \mathrm{rev} / \mathrm{min}$.

We include the possibility to run the designated compressors in recycle mode (also called bypass), i.e., redirecting some of the gas from the compressor output back to the input. Such a mode might be used when the gas flow demand is temporarily lower than the minimal compressor flow, and when it is not economically reasonable to shut the compressor down and restart it when the gas flow demand increases (e.g., a demand drop for one hour). 
Figure 3 Reciprocating compressor performance map for one particular rotation speed (in coordinates discharge pressure vs. suction pressure) (see online version for colours)

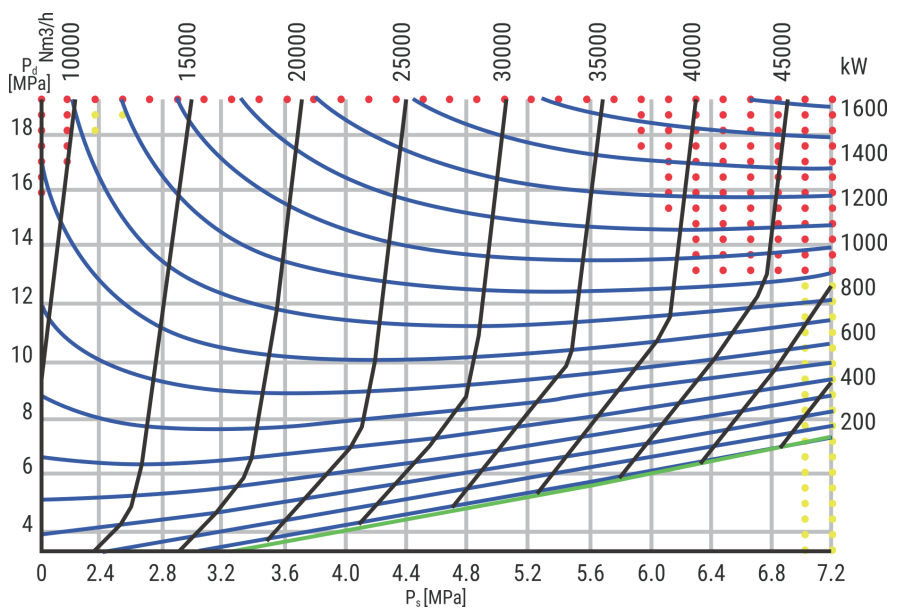

Notes: Red dots: forbidden operation, yellow dot: temporally allowed operation.

Figure 4 Turbocompressor characteristics map (in coordinates isentropic enthalpy increase vs. flow) (see online version for colours)

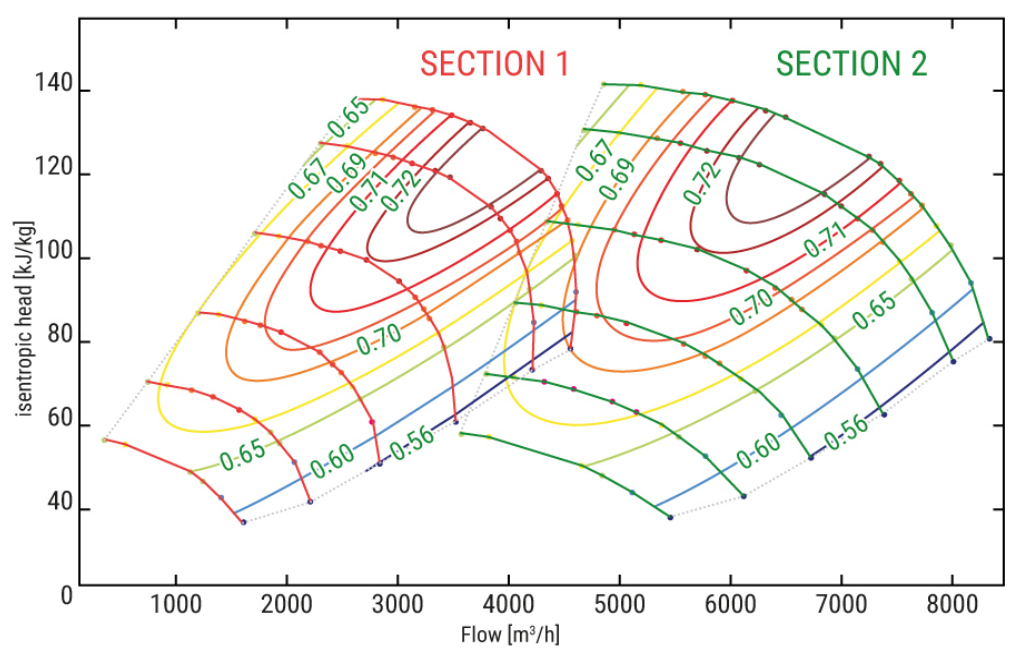

Note: The bold curves represent the rotations levels, the thin curves represent the efficiency levels.

Depending on the compressor type, we use different representations of their characteristics. In particular, for reciprocating compressors, the performance characteristics are usually presented by a family of curves relating output pressure to input pressure for a given shaft rotational speed (and for the shaft power) and a family of curves relating output pressure to input pressure for given gas flow and rotation speed, see Figure 3. The reason for this representation is dictated by the fact that for reciprocating compressors, the pressure ratio is practically independent of the gas composition and the input pressure. Similarly, for turbocompressors two families of 
curves are used. One family of curves relates the increase of the isentropic gas enthalpy to the gas flow for a given shaft rotational speed. The second family of curves relates the increase of the gas enthalpy to the gas flow for a given efficiency, see Figure 4 . These families of curves (the isentropic head) are convenient because there is little dependence of enthalpy increase on the conditions at the compressor inlet and on the gas composition. However it should be noted that different manufacturers use other representations, for example characteristic of polytropic efficiency. The compressor drive is described by the efficiency function $\eta^{\text {mech }}(r)$.

Besides the characteristics of compressors, we need to include the gas model to calculate gas thermodynamic properties. According to International Standard ISO 20765-1: (2005) the thermodynamic properties of the pipeline-quality natural gas depend on the gas composition and therefore they are characterised by component analysis calculations. To perform such calculations we developed a library containing several functions to calculate properties such as the increase of enthalpy or the work done to compress the gas in isentropic processes. The calculation of gas thermodynamic properties is also based on the norm International Standard ISO 20765-1: (2005).

\section{Theory and calculations}

The computations of dynamically changing parameters (e.g., flow, or compressor power) are performed with the resolution defined by the time-steps $t=1, \ldots, T$, asserting that the values of all parameters within the time-step are constant. A typical duration of the timestep is one hour. The index $i=1, \ldots, N$ denotes the compressor number. For each time-step $t$, the optimisation problem contains both continuous and discrete decision variables. The continuous variable denoted by $V_{i}(t)$ describes the flow through compressor $i$ for the timestep $t$. The discrete variables $m_{i}(t)$ 's represent the mode of compressor $i$ for the time-step $t$. Here, the mode could be either the compressor being on/off, in bypass mode, with sections connected in parallel or in serial (if such an option is available).

The total compressor operational cost is expressed by the formula

$$
\text { Total cost }=\sum_{t=1}^{T} \sum_{i=1}^{N}\left[C_{i, m_{i}(t)}^{\text {work }}\left(V_{i}(t), t\right)+C_{i, m_{i}(t-1), m_{i}(t)}^{\text {trans }}\right]
$$

where by $C_{i, m_{i}(t)}^{\text {work }}$ we denote the function representing the cost of running the compressor $i$, and by $C^{\text {trans }}$ the cost of switching the compressor from one configuration to another. The function $C_{i, m_{i}(t)}^{\text {work }}$ is calculated in Section 2.1, the function describing the transition costs $C^{\text {trans }}$ is specified by the storage operator. This function includes the cost of compressor start-up. We skip all constant costs, or costs independent of compressor configuration and power usage, since our purpose is to lower the costs generated directly by the compressors operation. However, we do include the service costs depending on the compressor's working hours.

If we had had the ability to freely switch the configuration of the compressor unit (without generating any extra costs) between the consecutive time-steps, the optimisation could have been performed independently for each time-step, and we would only need to optimise locally. This is however not the case in the real world where direct switching 
from a certain configuration to another might be not possible, or it generates extra costs. For example restarting a compressor takes time and energy, both of which add to cost. Therefore, the optimisation has to be considered globally, i.e., we need to determine globally the values of each $V_{i}(t)$ and each $m_{i}(t)$ for which the total cost is minimised. In our case the optimisation procedure can be significantly simplified by performing it in three consecutive phases (calculation of the cost function and the cost limits, solving the continuous optimisation problem, and solving the discrete optimisation problem), each considered as separate sub-problems. Phase one of the calculations is described in details in Section 2.1. Here, we focus on the second phase, i.e., the determination of the continuous variables. We use the fact that for any given set of $m_{i}(t)$ values, we can minimise the sum

$$
\sum_{t=1}^{T} C^{w o r k}\left(t, V_{1}(t), V_{2}(t), \ldots, V_{N}(t)\right) \equiv \sum_{t=1}^{T} \sum_{i=1}^{N} C_{i, m_{i}(t)}^{w o r k}\left(V_{i}(t), t\right)
$$

by minimising each term $C^{\text {work }}\left(t, V_{1}(t), V_{2}(t), \ldots, V_{N}(t)\right)$ independently with the constraints

$$
\sum_{i=1}^{N} V_{i}(t)=V^{t o t}(t), \quad \text { and } V_{i, m_{i}(t)}^{\min } \leq V_{i}(t) \leq V_{i, m_{i}(t)}^{\max },
$$

That is the sum all of flows gives the contracted total flow, and we do not exceed the flow limits of each compressor. We denote the minimising solution by $\tilde{V}_{i, m_{i}(t)}(t)$. In particular, the minimising solution is found by performing SQP optimisation for each time-step $t$ and for each compressors configuration $m_{i}(t)$. To this end, we need to calculate the cost function $C^{\text {work }}$ and the flow limits $V_{i, m_{i}(t)}^{\min }$ and $V_{i, m_{i}(t)}^{\max }$ for each compressor $i$ (see the algorithm flow chart in Figure 5, the first three loops). The details on how to compute the cost function and the flow limits are presented in Section 2.1. These details depend on the compressor type and its operational mode. At this point in the process we can determined the optimal distribution of flows for any mode set $\left\{m_{i}(t) \mid i=1, \ldots, N, t=1, \ldots, T\right\}$. Once we determine the set of optimal flows those solutions for all possible combinations of $m_{i}(t)$ 's for each single $t$, the total cost can be expressed by the formula containing only the discrete variables $m_{i}(t)$.

$$
\text { Total cost }=\sum_{t=1}^{T} \sum_{i=1}^{N}\left[C_{t, i, m_{i}(t)}^{\text {work }}+C_{i, m_{i}(t-1), m_{i}(t)}^{\text {trans }}\right]
$$

This is the quality indicator used in the third phase of the calculations.

Now we proceed to the third phase of the calculations where we deal with the problem of determining the optimal decision trajectory of the discrete variables $m_{i}(t)$. More precisely, for each $t$ and each compressor $i$, we determine the optimal discrete modes (e.g., off, on parallel, on-serial) assuming that for each available mode we had calculated the optimal flow and related operation costs. As will be shown, this kind of problem is solved by dynamic programming. To apply this method the following condition has to be satisfied: If the decision trajectory $m(t), m(t+1), \ldots, m(T)$ for a process with $T-t$ steps represents the optimal trajectory, then the decision trajectory $m(t+1)$, $m(t+2), \ldots, m(T)$ shall also be an optimal trajectory for the process with $T-(t+1)$ steps and with the initial mode $m(t)$. 
Figure 5 Algorithm flow

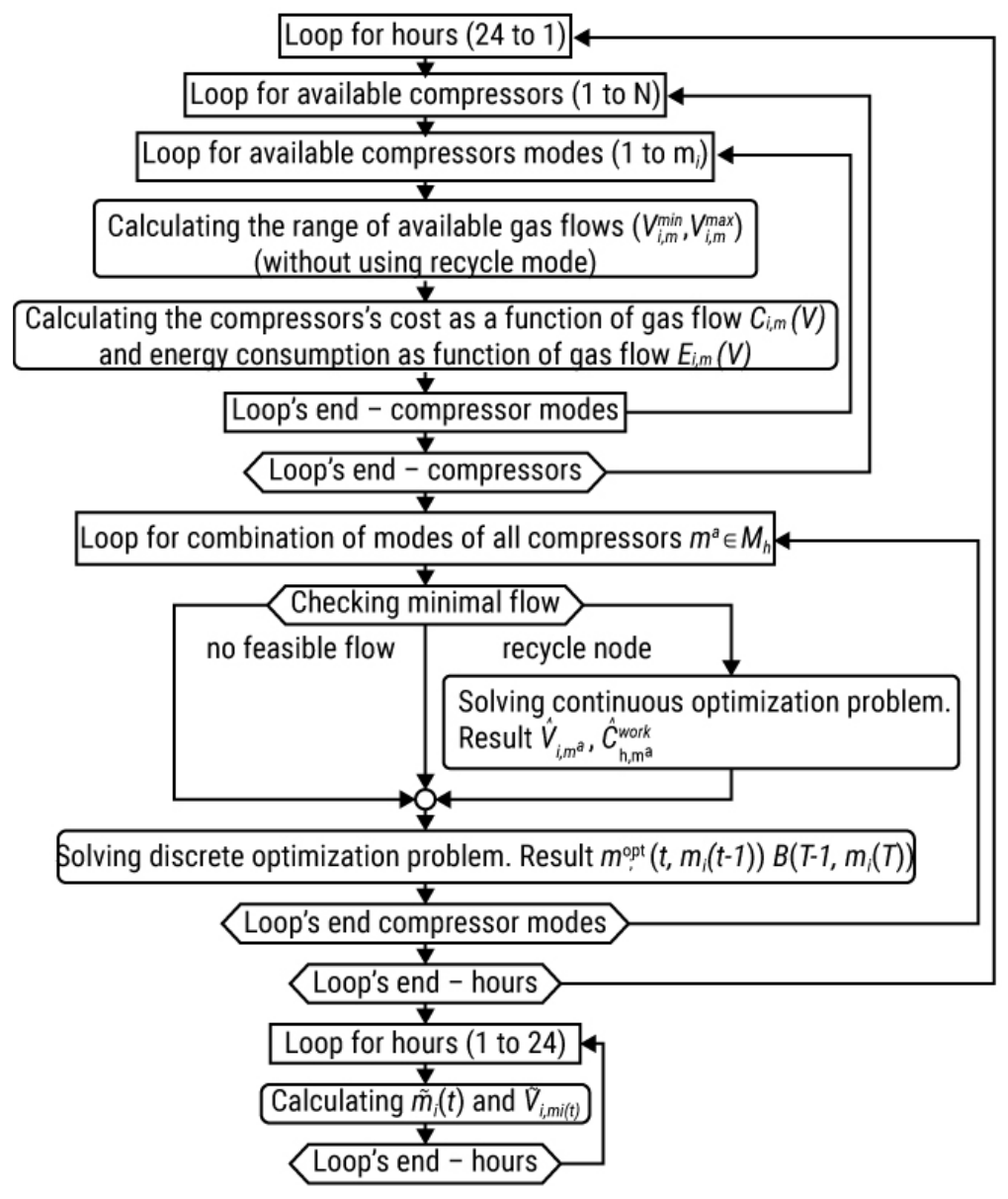

In our case the following property holds: let us consider the time-step $t=T-1$ and some specific modes $m_{i}(T-1)$. We can easily check which mode in the final step $t=T$ should be chosen by calculating the contribution into the total cost for all values of $m_{i}(t)$, see equarion (4). For each $i$ we therefore determine the optimal mode $m_{i}^{\text {opt }}\left(T \mid m_{i}(T-1)\right)$ in the final step $t=T$ on the condition that the modes $m_{i}(T-1)$ in the current step $t=T-1$ are known, see Figure 6. The previous calculation is repeated for all $m_{i}(T-1)$, and we thus obtain the values $m_{i}^{\text {opt }}\left(T \mid m_{i}(T-1)\right)$ and the associated costs. The calculations performed for the time-step $t=T-1$ can be easily generalised. Let us denote by $B\left(t, m_{i}(t)\right)$ the Bellman function, i.e., the aggregated costs for the optimal trajectory that starts with some given mode $m_{i}(t)$. Therefore, $B\left(T-1, m_{i}(T-1)\right)$ is the cost of work at $t=T$ plus the cost of transition between the modes $m_{i}(T-1)$ and the modes $m_{i}^{o p t}(T)$, where $m_{i}^{o p t}(T)$, represent the modes of the optimal trajectory starting with $m_{i}(T-1)$. If we know $B\left(T-1, m_{i}(t)\right)$ for any $m_{i}(T-1)$, then we can similarly determine the value of $m_{i}^{o p t}\left(T-1 \mid m_{i}(T-2)\right)$ once we initiate the trajectory with any specific value of $m_{i}(T-2)$. 
Generalising, for any given $t$ and $m_{i}(t)$ we can determine each $m_{i}^{o p t}(t+1)$, on the condition that we are able to compute $B\left(t, m_{i}(t)\right)$, which represents the aggregated costs of the optimal trajectory starting with the modes $\left.m_{i}(t+1)\right)$ at $t+1$. Calculation of $B\left(t, m_{i}(t)\right)$ from $B\left(t+1, m_{i}(t+1)\right)$ is straightforward. By induction, we can determine the function $m_{i}^{o p t}\left(t \mid m_{i}(t-1)\right)$ for $t=1, \ldots T$. In the third phase of the optimisation procedure, this function allows us to recursively calculate the solutions $\tilde{m}_{i}(1)=m_{i}^{o p t}\left(1, m_{i}(0)\right)$, $\tilde{m}_{i}(2)=m_{i}^{o p t}\left(2, m_{i}(1)\right), \ldots$, where the modes $m_{i}(0)$ represent the initial conditions. In this way we obtain the optimal decision trajectory.

Figure 6 The computational step necessary to determine the optimal trajectory for discrete variables for the system containing two compressors with modes off/on-parallel/on-serial (see online version for colours)

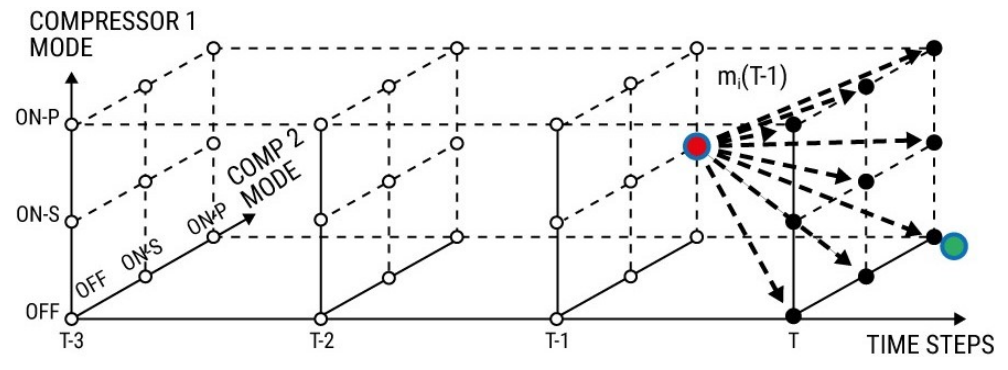

Notes: Starting from the given modes $m_{i}(T-t)$ at $t=T-1$ (red dot), we calculate the aggregated costs for all possible changes of configuration while moving from steps $T-1$ to $T$. Hence, we determine the optimal modes $m_{i}^{o p t}\left(T \mid m_{i}(T-1)\right)$ 's at $t=T$ (green dot), on the condition that at $t=T-1$ the modes were $m_{i}(T-1)$.

We further note that, if for certain modes $m_{i}(t)$ at time $t$ we have

$$
\sum_{i=1}^{N} V_{i, m_{i}(t)}^{\min } \geq V^{t o t}(t)
$$

i.e., the total flow is too small to allow for using the configuration described by the modes $m_{i}(t)$, we can still proceed by configuring one of the compressors into the 'recycle mode', if such an option is available. Let $r$ be the index of a compressor which can work in the 'recycle mode' (if there are many such compressors, we choose the one with the highest value of $\left.V_{i, m_{i}(t)}^{\min }\right)$. This compressor can be configured into the 'recycle mode' if we have

$$
\sum_{i \neq r, i=1 \ldots N} V_{i, m_{i}(t)}^{\min } \geq V^{t o t}(t)
$$

and consequently the solution to the sub-problem is

$$
\begin{array}{ll}
\tilde{V}_{i, m_{i}(t)}=V_{i, m_{i}(t)}^{\min }(t) & i \neq r \\
\tilde{V}_{i, m_{i}(t)}=V^{t o t}(t)-\sum_{i \neq r} V_{i, m_{i}(t)}(t) & i=r .
\end{array}
$$


If equation (5) does not hold then the configuration described by the modes $m_{i}(t)$ cannot be supported, i.e., the minimal total flow of one of the compressors working in the 'recycle mode' is still too large.

\subsection{Compressor work cost function and flow limits}

In order to determine the optimal values of the continuous variables in equations (2) and (4), i.e., to distribute the flows in an optimal way between compressors for the specific configuration modes $m_{i}(t)$, we need to know the form of the cost function which appears in equation (1). As the form of the cost function depends on the compressor type and the mode, we will consider separately the following cases:

- reciprocating compressor

- turbocompressor in parallel mode

- turbocompressor in serial mode.

We assume that the compressor which is switched off does not generate any cost. Since, in this section, we perform the calculations for the specific compressor, and for the given time-step, we do not explicitly display indices $i$ and $t$. Apart from the cost function, we need to determine the domain of allowed flow values, for each type of compressors.

\subsubsection{Reciprocating compressor}

The reciprocating compressor is characterised by the two functions $\mathcal{P}_{V}^{d}\left(p^{s}, V^{N}, r\right)$ and $\mathcal{P}_{P}^{d}\left(p^{s}, P, r\right)$, both of which represent output pressure but with respect to different inputs. In particular, both functions depend on the input pressure $p^{s}$ and the rotational speed $r$, however, $\mathcal{P}_{V}$ depends on the normalised flow $V^{N}$ and $\mathcal{P}_{P}$ depends on the shaft power $P$.

The compressor drive is described by the maximum power function $P^{\max }(r)$ and efficiency function $\eta^{\text {mech }}(r)$. If the compressor is driven by a gas turbine, then both of these functions also depend on the ambient temperature $T^{a m b}$. If the compressor is driven by an electric motor, these functions are usually constant. We do not display explicitly in the notation the dependence on the ambient temperature, which for each step of computation is assumed to be a constant parameter.

We also need to consider the following parameters:

c gas composition

$T^{\infty} \quad$ gas temperature at the compressor inlet

$r_{\min }$ and $r_{\max }$ minimum and the maximum shaft rotation speed (dictated by the limits of the compressor, and the limits of the drive),

$F \quad$ domain in $\mathbb{R}^{2}$ containing the values $\left(p^{s}, p^{d}\right)$ in which the compressor can operate for a given rotational speed $r$

$\Delta p^{c} \quad$ pressure drop in the gas cooler 
$W$

energy price (e.g., price of gas or electricity used to power the compressor), which can be different for each hour within the optimisation horizon.

In order to determine the cost function, and the maximal and minimal flow limits, we need to calculate the minimal shaft power $P_{\min }$ at which the compressor can work for given values of $p^{d}$ and $p^{s}$. To end of this, we solve the equation

$$
\mathcal{P}_{P}^{d}\left(p^{s}, P_{\min }, r_{\max }\right)=p^{d}+\Delta p^{c}
$$

for $P_{\min }$.

If $P_{\max }\left(r_{\min }\right)<P_{\min }$, then the drive is not able to supply a rotational moment high enough to run the compressor at rotation speed $r_{\min }$. In such a case we need to operate at some higher rotation speed $\sim r_{\min }$ that can be handled by the drive, taking into account the compressor characteristics. To this end, we solve the equation

$$
\mathcal{P}_{P}^{d}\left(p^{s}, P^{\max }\left(\tilde{r}_{\text {min }}\right), \tilde{r}_{\text {min }}\right)=p^{d}+\Delta p^{c}
$$

for $\tilde{r}_{\text {min }}$, and we use the new value of $\tilde{r}_{\text {min }}$ as the minimal value of rotation speed which can be handled by the compressor with the drive. We need obviously to check whether $\tilde{r}_{\min }<r_{\max }$. If this condition is not satisfied, then there is no solution for the given mode and values of input and output pressure.

In the next step, we calculate the minimal and the maximal value of the normalised gas flow, $V_{\min }^{N}$ and $V_{\max }^{N}$, by solving the equation

$$
\mathcal{P}_{V}^{d}\left(p^{s}, V^{N}, r\right)=p^{d}+\Delta p^{c}
$$

for $V^{N}$ with $r=r_{\min }$ and $r=r_{\max }$, respectively.

Subsequently, we determine the physical gas flow limits from the normalised flow values

$$
V_{\min }=V^{p h y s}\left(V_{\min }^{N}, p^{s}, T^{s}, c\right)
$$

and similarly for $V_{\max }$. If we have an option to run the compressor in the 'recycle mode' we can still operate with the flow $V<V_{\min }$, in this case the actual flow through the compressor is $V_{\min }$, but we need to divert the flow $V_{\min }-V$ through the bypass installation. In the range $V_{\min } \leq V \leq V_{\max }$ we can determine the normalised flow from $V^{N}=V^{n o r m}\left(c, p^{s}, T^{s}, V\right)$. The function $V^{n o r m}$ is based on state equation of the real gas, where the compressibility factor is calculated according to International Standard ISO 20765 (2005) norm, and by solving (9) for rotational speed $r$. Having the value of $r$, we calculate the shaft power $P$ from equation

$$
\mathcal{P}_{P}^{d}\left(p^{s}, P, r\right)=p^{d}+\Delta p^{c} .
$$

The cost of driving the gas compressor (the function needed to solve the first phase of the optimisation problem) is given then by 


$$
C=w \frac{P}{\eta(r)} \Delta t,
$$

where $\Delta t$ is the duration of the time-step.

If we run the compressor in the 'recycle mode', it still compresses $V_{\min }$ amount of gas, therefore we use equation (12) with the substitution $P \rightarrow P_{\min }$.

\subsubsection{Turbocompressor}

In order to determine the available flow limits and to calculate the cost function for turbocompressors, we need to know the form of the following functions.

$\Delta h^{s}(r, V) \quad$ increase of isentropic enthalpy (per unit mass) as a function of shaft rotation speed and physical input gas flow at suction conditions

$\eta(r, V) \quad$ isentropic efficiency

$V^{\min }(r)$ and $V^{\max }(r)$ minimal and maximal gas flow at given shaft rotation speed, determined by the surge line, and the choke line respectively.

We also need to take into account the mechanical efficiency of the compressor, and the pressure drop in the cooler, denoted by $\Delta p^{c}$. For the sake of simplicity we will consider only the most common case, in which two sections of the turbocompressor are driven by the same shaft. The results can easily be generalised to the case with more than two sections. We use two sets of parameters and functions, with a subscript (I or II) indexing the compressor section. The parameters that are common to both sections are the rotation speed mechanical limits $r_{\min }$ and $r_{\max }$, as both sections are driven by the same shaft. We note that $V_{\max }\left(r_{\max }\right)$ and $\left.V_{\min }\left(r_{\min }\right)\right)$ do not simply define us the range of feasible flow, it may happen that we are not able to operate at mechanical limits due to improper thermodynamic properties of our system. The further details depend on whether the sections are connected in parallel or serial mode. The mechanical efficiency of the drive $\eta^{\text {mech }}$ is included in the final calculation of the cost function.

\subsubsection{Turbocompressor in parallel mode}

The cost function for a turbocompressor in parallel mode, Figure 7, is calculated using isentropic performance curves. In the first step we determine the sustainable range of flows $\left\langle\tilde{V}_{\min }, \tilde{V}_{\max }\right\rangle$ for given gas parameters, assuming that the 'recycle mode' is not used. To this end, we calculate the increase of isentropic enthalpy per unit mass for Section 1 of the compressor

$$
\Delta h_{I}=\Delta h\left(c, p^{s}, p^{d}+\Delta p_{I}^{c}, T^{s}\right) .
$$

Using the compressor characteristics $\Delta h_{s}$, we define the lower limit of enthalpy by

$$
\Delta h_{I}^{L}=\Delta h_{I}^{s}\left(r_{\min }, V_{I}^{\min }\left(r_{\min }\right)\right) .
$$


Figure 7 Structure of a compressor in parallel mode

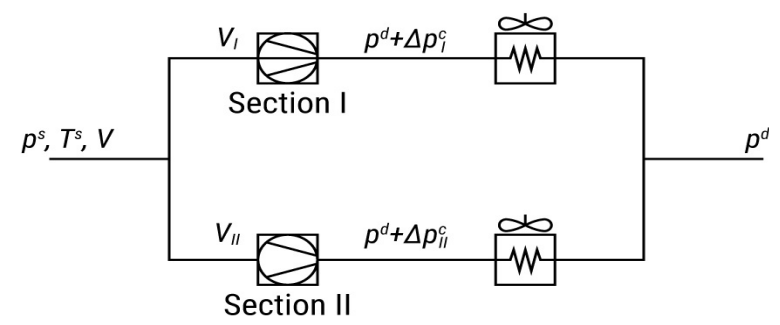

If $\Delta h^{I}>\Delta h_{I}^{L}$ than the minimal sustainable rotation speed $\tilde{r}_{\text {min }}$ is determined from the following equation:

$$
\Delta h_{I}=\Delta h_{I}^{s}\left(\tilde{r}_{\min }, V_{I}^{\min }\left(\tilde{r}_{\min }\right)\right),
$$

and the minimal sustainable flow is calculated as

$$
\tilde{V}_{\min }=V_{I}^{\min }\left(\tilde{r}_{\min }\right) .
$$

If $\Delta h^{I}>\Delta h_{I}^{L}$ then we can operate at $r_{\min }$ with the flow $\tilde{V}_{\min }>V_{\min }\left(r_{\min }\right)$, which we find by solving the equation

$$
\Delta h_{I}^{s}=\Delta_{I}^{s}\left(r_{\min }, \tilde{V}_{I}^{\min }\right) .
$$

In an analogousway we proceed to determine the sustainable values of maximal rotation speed $\tilde{r}_{\max }$ and the maximal possible flow $\tilde{V}_{\max }$. Instead of equation (14), we need to calculate

$$
\Delta h_{I}^{U}=\Delta h_{I}^{s}\left(r_{\max }, V_{I}^{\max }\left(r_{\max }\right)\right)
$$

and to check whether $\Delta h_{I}>\Delta h_{I}^{U}$. We obtain the values of $\tilde{r}_{I I}^{\min }, \tilde{r}_{I I}^{\max }, \tilde{V}_{\min }^{I I}, \tilde{V}_{\max }^{I I}$ by means of the same calculations performed for Section 2 of the compressor. If $\tilde{r}_{I}^{\min }<\tilde{r}_{I I}^{\min }$ then Section 2 of the compressor reaches its minimal rotation speed limit first, therefore the rotation speed is restricted from below by

$$
\tilde{r}^{\min }<\tilde{r}_{I I}^{\min } .
$$

In this case, $\tilde{V}_{I}^{\min }$ is calculated by solving

$$
\Delta h_{I}=\Delta h_{I}^{s}\left(\tilde{r}^{\min }, \tilde{V}_{I}^{\min }\right) .
$$

We proceed similarly if $\tilde{r}_{I I}^{\min }<\tilde{r}_{I}^{\min }$. The values of $\tilde{r}^{\max }, \tilde{V}_{I}^{\max }, \tilde{V}_{I}^{\max }$ are determined in an analogous way.

Next, we calculate the mechanical work done by each section of the compressor for the maximal shaft rotation speed for the specific gas composition $c$ using

$$
P_{l \text { mech }}=P_{\text {mech }}\left(c, p^{s}, p^{d}+\Delta p^{c}, T^{s}, \eta l\left(\tilde{r}_{\max }, \tilde{V}_{l}^{\max }\right), \tilde{V}_{l}\right),
$$

where $l$ numbers the compressor section. If 


$$
P_{\text {mech I }}+P_{\text {mech II }}>P_{\max }\left(\tilde{r}^{\max }\right)
$$

Then, due to insufficient drive power, we cannot operate at previously calculated values of the maximal flows $V_{I}^{\max }$ and $V_{I I}^{\max }$. In this case we need to recalculate the values of $\tilde{r}^{\max }, V_{I}^{\max }$ and $V_{I I}^{\max }$ from the following equations.

$$
\left\{\begin{array}{l}
P_{\text {mech }}\left(c, p^{s}, p^{d}+\Delta p_{I}^{c}, T^{s}, \eta_{I},\left(r, V_{I}\right), V_{I}\right) \\
\quad+P_{\text {mech }}\left(c, p^{s}, p^{d}+\Delta p_{I I}^{c}, T^{s}, \eta_{I I},\left(r, V_{I I}\right), V_{I I}\right)=P^{\max }(r) \\
\Delta h_{I}^{s}=\Delta h_{I}^{s}\left(r, V_{I}\right) \\
\Delta h_{I I}^{s}=\Delta h_{I I}^{s}\left(r, V_{I I}\right)
\end{array}\right.
$$

When the flow $V$ is within the range $\left[V_{I}^{\min }+V_{I I}^{\min }, V_{I}^{\max }+V_{I I}^{\max }\right]$ he compressor can work in a normal mode, without recycling. In this case the flows $V_{I}, V_{I I}$ and the rotational speed $r$ can be calculated as follows:

$$
\left\{\begin{array}{l}
\Delta h_{I}^{s}=\Delta h_{I}^{s}\left(r, V_{I}\right) \\
\Delta h_{I I}^{s}=\Delta h_{I I}^{s}\left(r, V_{I I}\right) \\
V_{I}+V_{I I}=V_{i}
\end{array}\right.
$$

The power cost for the compressor in question is finally given by

$$
\begin{aligned}
C^{\text {work }}= & \frac{w}{\eta\left(r, T^{a m b}\right)} \cdot P_{\text {mech }}\left(c, p^{s}, p^{d}+\Delta p_{I}^{c}, T^{s}, \eta_{I}\left(r, V_{I}\right), V_{I}\right)+ \\
& +P_{\text {mech }}\left(c, p^{s}, p^{d}+\Delta p_{I}^{c}, T^{s}, \eta_{I I}\left(r, V_{I I}\right), V_{I I}\right) .
\end{aligned}
$$

\subsubsection{Turbocompressor in serial mode}

The parameters describing a compressor configured into serial mode are the same as in parallel mode, except for additional parameters $T_{m}$ and $p_{m}$, see Figure 8 for definitions. Due to the presence of the intermediate pressure and temperature, $p_{m}$ and $T_{m}$, in order to solve the first phase of the optimisation problem, i.e., to determine the optimal flow for given modes $m^{i}(t)$, we need to include the following constraints:

$$
\begin{aligned}
& \Delta h_{I}^{s}(r, V)=\Delta h\left(c, p^{s}, p^{m}+\Delta p_{I}^{c}, T^{s}\right), \\
& \Delta h_{I I}^{s}\left(r, V^{\text {recalc }}\left(V, T^{s}, p^{s}, T^{m}, p^{m}\right)\right)=\Delta h\left(c, p^{m}, p^{d}+\Delta p_{I I}^{c}, T^{m}\right),
\end{aligned}
$$

and

$$
\begin{aligned}
& r_{\min } \leq r \leq r_{\max }, \\
& V_{I}^{\min }(r) \leq V \leq V_{I}^{\max }(r), \\
& V_{I I}^{\min }(r) \leq V^{\text {recalc }}\left(V, T^{s}, p^{s}, T^{m}, p^{m}\right) \leq V_{I I}^{\max }(r) .
\end{aligned}
$$

where the function $V^{\text {recalc }}\left(V, T^{\prime}, p^{\prime}, T^{\prime \prime}, p^{\prime \prime}\right)$ gives us the physical flow at temperature $T^{\prime \prime}$ and pressure $p^{\prime \prime}$, from the value $V$ at temperature $T^{\prime}$ and pressure $p^{\prime}$. 
Figure 8 Structure of a compressor in serial mode

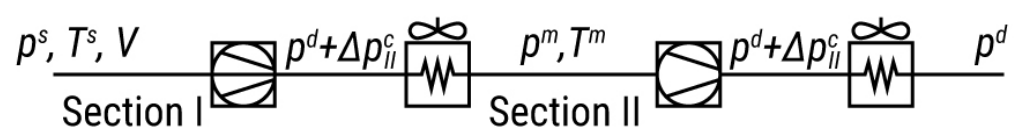

Equality constraints (22) and (23) limit the dimension of the parameter space described by $\left(V, r, p^{m}\right)$ to one dimension. Therefore, exactly one inequality constraint will be relevant to finding a solution to the optimisation problem. This will be the inequality that is violated 'before' the others. Since applying equations (22) and (23) yields a positive correlation between $r$ and $V$, we can ignore the inequalities

$$
\begin{aligned}
& r \leq r_{\max } \\
& V \leq V_{I}^{\max }(r) \\
& V^{\text {recalc }}\left(V, T^{s}, p^{s}, T^{m}, p^{m}\right) \leq V_{I I}^{\max }(r) .
\end{aligned}
$$

and only one of three cases can occur:

$$
\begin{array}{ll}
\text { a } & r_{\min }=r \\
\text { b } & V_{I}^{\min }=V \\
\text { c } & V_{I I}^{\min }(r)=V^{\text {recalc }}\left(V, T^{s}, p^{s}, T^{m}, p^{m}\right) .
\end{array}
$$

In the optimisation problem we use the following three-step procedure to verify the above cases:

1 if the first case holds, we solve the following set of equations

$$
\left\{\begin{array}{l}
\Delta h_{I}^{s}\left(r_{\min }, V\right)=\Delta h\left(c, p^{s}, p^{m}+\Delta p_{I}^{c}, T^{s}\right) \\
\Delta h_{I I}^{s}\left(r_{\min }, V^{\text {recalc }}\left(V, T^{s}, p^{s}, T^{m}, p^{m}\right)\right)=\Delta h\left(c, p^{m}, p^{d}+\Delta p_{I I}^{c}, T^{m}\right)
\end{array}\right.
$$

with respect to $V$ and $p^{m}$ and denote the solution as $\tilde{V}_{\min }$ and $\tilde{p}_{\min }^{m}$.

2 subsequently, we check the second constraint

$$
V_{I}^{\min }\left(\tilde{r}_{\min }\right) \leq \tilde{V}_{\min }
$$

If it is violated, then the minimum rotation speed and the gas flow can be found by solving the equations

$$
\left\{\begin{array}{l}
\Delta h_{I}^{s}\left(r, V_{I}^{\min }(r)\right)=\Delta h\left(c, p^{s}, p^{m}+\Delta p_{I}^{c}, T^{s}\right) \\
\Delta h_{I I}^{s}\left(r, V^{\text {recalc }}\left(V_{I}^{\min }(r), T^{s}, p^{s}, T^{m}, p^{m}\right)\right)=\Delta h\left(c, p^{m}, p^{d}+\Delta p_{I I}^{c}, T_{m}\right)
\end{array}\right.
$$

with respect to $r$ and $p^{m}$. We denote the solution as $\tilde{r}_{\text {min }}$ and $\tilde{p}_{\min }^{m}$. In this case we have $\tilde{V}_{\min }=V_{I}^{\min }\left(\tilde{r}_{\min }\right)$. 
3 Finally, we check the third constraint

$$
V_{I I}^{\min }\left(\tilde{r}_{\min }\right) \leq V^{\text {recalc }}\left(\tilde{V}_{\min }, T^{s}, p^{s}, T^{m}, \tilde{p}_{\min }^{m}\right) .
$$

If it is violated, then the third case holds and the minimum rotation speed and the gas flow can be found by solving the equations.

$$
\left\{\begin{array}{l}
\Delta h_{I}^{s}(r, V)-\Delta h\left(c, p^{s}, p^{m}+\Delta p_{I}^{c}, T^{s}\right) \\
\Delta h_{I I}^{s}\left(r, V^{\text {recalc }}\left(B, T^{s}, p^{s}, T^{m}, p^{m}\right)\right)=\Delta h\left(c, p^{m}, p^{d}+\Delta p_{I I}^{c}, T^{m}\right) \\
V_{I I}^{\min }(r)=V^{\text {recalc }}\left(v, T^{s}, p^{s}, T^{m}, p^{m}\right)
\end{array}\right.
$$

with respect to $V, r$, and $p^{m}$. We denote the solution as $\tilde{V}_{\min }, \tilde{r}_{\min }$ and $\tilde{p}_{\min }^{m}$.

The values of $\tilde{V}_{\max }, \tilde{r}_{\max }$ and $\tilde{p}_{\max }^{m}$ are determined in an analogous way. In the next phase we calculate the mechanical work done in each compressor section for the maximal shaft rotation speed given by

$$
\begin{aligned}
& P_{I}^{\max }=P_{\text {mech }}\left(c, p^{s}, \tilde{p}_{\max }^{m}+\Delta p_{I}^{c}, T^{s}, \eta_{I}\left(\tilde{r}_{\max }, \tilde{V}_{\max }\right), \tilde{V}_{\max }\right) \\
& P_{I I}^{\max }=P_{\text {mech }}\left(\begin{array}{l}
c, \tilde{p}_{\max }^{m}, p^{d}+\Delta p_{I I}^{c}, T^{m} \eta_{I I} \\
\left(\tilde{r}_{\max }, V^{\text {recalc }}\left(\tilde{V}_{\max }, T^{s}, p^{s}, T^{m}, p_{m}\right)\right) \\
V^{\text {recalc }}\left(\tilde{V}_{\max }, T^{s}, p^{s}, T^{m}, p_{m}\right)
\end{array}\right)
\end{aligned}
$$

If $P_{I}^{\max }+P_{I I}^{\max }>P_{\max }\left(r_{\max }, T^{a m b}\right)$ then we determine the values of $\tilde{V}_{\max }, p_{\max }^{m}$ and $r_{\max }$ from solving the following set of equations

$$
\begin{aligned}
& P_{\text {mech }}\left(c, p^{s}, p^{m}+\Delta p_{I}^{c}, T^{s}, \eta_{I}(r, V), V\right) \\
& +P_{\text {mech }}\left(c, p^{m}, p^{d}+\Delta p_{I I}^{c}, T^{m}, \eta_{I I}\left(r, V^{\text {recalc }}\left(V, T^{s}, p^{s}, T^{m}, p^{m}\right)\right),\right. \\
& \left.\quad V^{\text {recalc }}\left(V, T^{s}, p^{s}, T^{m}, p^{m}\right)\right)=P^{g \max }\left(r, T^{a m b}\right) \\
& \Delta h_{I}^{s}(r, V)=\Delta h\left(c, p^{s}, p^{m}+\Delta p_{I}^{c}, T^{s}\right) \\
& \Delta h_{I I}^{s}\left(r, V^{\text {recalc }}\left(V, T^{s}, p^{s}, T^{m}, p^{m}\right)\right)=\Delta h\left(c, p^{m}, p^{d}+\Delta p_{I I}^{c}, T^{m}\right)
\end{aligned}
$$

with respect to $V, p^{m}$, and $r$.

When the flow is within the range $\left[\tilde{V}_{\min }, \tilde{V}_{\max }\right]$ the compressor can work in normal mode without the bypass. For lower flows the bypass has to be used.

To calculate the cost of gas compression, $C^{\text {work }}$, for the input flow $V$, we need to solve the following set of equations

$$
\begin{aligned}
& \Delta h_{I}^{s}\left(r, V_{i}\right)=\Delta h\left(c, p^{s}, p^{m}+\Delta p_{I}^{c}, T^{s}\right) \\
& \Delta h_{I I}^{s}\left(r, V^{\text {recalc }}\left(V_{i}, T^{s}, p^{s}, T^{m}, p^{m}\right)\right)=\Delta h\left(c, p^{m}, p^{d}+\Delta_{I I}^{c}, T^{m}\right)
\end{aligned}
$$

with respect to $p^{m}$ and $r$. The power cost is obtained as 


$$
\begin{aligned}
C_{i}= & \frac{w}{\eta^{\text {mech }}\left(r, T^{\text {amb }}\right)} \cdot\left(P^{\text {mech }}\left(c, p^{s}, p^{m}+\Delta p_{I}^{c}, T^{s}, \eta_{I}\left(r, V_{i}\right), V_{i}\right)\right. \\
& +P^{\text {mech }}\left(\begin{array}{l}
c, p^{m}, p^{d}+\Delta p_{I I}^{c}, T^{m}, \eta_{I I}\left(r, V^{\text {recalc }}\left(V_{i}, T^{s}, p^{s}, T^{m}, p^{m}\right)\right) \\
V^{\text {recalc }}\left(V^{i}, T^{s}, p^{s}, T^{m}, p^{m}\right)
\end{array}\right)
\end{aligned}
$$

\subsection{Computational accuracy}

The methodologies and assumptions used here impact the accuracy of the results. The main factors influencing the accuracy include:

- The accuracy of the forecast of input parameters, such as inlet and outlet pressure, temperature forecast, and gas composition forecasting, have a crucial impact on the calculation quality. Unfortunately, there are no clear mechanisms for precise forecast of input parameters.

- The assumption of constant pressure drop in the cooler. With a properly taken pressure drop value, the impact of this simplification is rather small and can be ignored.

- The accuracy of the characteristics of devices. The characteristics provided by the compressor suppliers are used. Those characteristics can degrade over time due to device usage. Since updating characteristics is a complicated process, it might happen that the algorithm is fed with imprecise characteristics.

- The errors in numerical solving of equations and numerical optimisation methods. The methods used to solve equations allow for control of numerical error and as such the methods were configured to produce the solutions without significant numerical errors impacting on accuracy.

- The application of piecewise linear functions simplifies calculations and implementation. The impact on calculation accuracy (with the assumption that a specific number of pieces are used) is marginal.

- The time needed to change configuration from serial to parallel and vice versa. Precise implementation requires a large effort with regards to conceptual work and programming. We approximate by neglecting the configuration change time, if for example, during the change the compressor will be stopped for 15 minutes, then the operator will be responsible for increasing flow for the next few hours to meet the daily contract. The configuration depends mostly on storage pressure which does not change that frequently, and therefore the simplification can be accepted.

\section{Results}

In this section we present exemplary results showing the details of the operation of the proposed method, when applied to controlling the real compressor site. 
Figure 9 The compressor site with five compressor units working in parallel (stage 1), and two additional compressors (stage 2) optionally connected to increase the output pressure (see online version for colours)

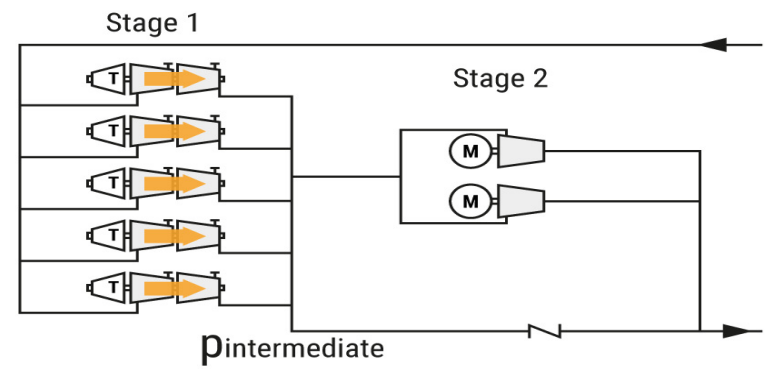

The discussed compressor site consists of five compressor units working in parallel with an option to connect a second stage of two compressor units to increase the output pressure (the cascade topology), see Figure 9. In this case, when the compressors in the second block are used, we need to generalise the presented formalism. We treat the cascade topology by breaking the optimisation problem into two sub-problems as follows: We treat the value $p_{\text {interstage }}$ of the pressure in the pipeline connecting both blockes of compressors as an unknown variable, see Figure 9. For any specific value of $p_{\text {interstage }}$ we can separately perform the optimisation for both stages. Therefore, we can proceed to finding the value of $p_{\text {interstage }}$ for the optimal configuration using a fast converging scalar minimisation algorithm [such as Brent's (1973) method], looking for the minimum of the sum of operational costs for both stages. It the discussed example, there were no coolers between both stages of compressors, therefore in the configuration shown in Figure 8, $p_{I I}^{s}=p_{I}^{d}$.

For the given flow trajectory $V^{\text {tot }}(t)$ we used the discussed method to determine the trajectory of continuous $\left(V_{i}(t)\right.$ 's) and discrete $\left(m_{i}(t)\right.$ 's) variables, and calculated the operational costs at each of the time-step $t$. For the purpose of method evaluation, we also performed a simulation calculating the operational costs for the case a) when the compressor station was controlled by the human operator (manual control), and b) when the compressor station was controlled by the proposed method (automatic control). The analysed data origin from the real gas storage system, however in order to fulfil the confidentiality requirements, the presented values in the plots were rescaled by an overall randomly chosen constant. The trajectories of the continuous variables, i.e., the flow for each of compressors, are presented in Figure 10 and Figure 11, respectively. We can clearly see more frequent switches of flow division for the automatic control in days 55-90, see Figure 11.

The comparison of the operational costs for both operational strategies (manual operation versus proposed automatic method), over a six month period, is shown in Figure 13. At each month, application of the proposed method brings savings, the total savings during the studied period were $\approx 5.43 \%$. 
Figure 10 Flow division (the flow for each compressor $C 1-C 5$, as a function of time) for the manual control of the compressor units (see online version for colours)

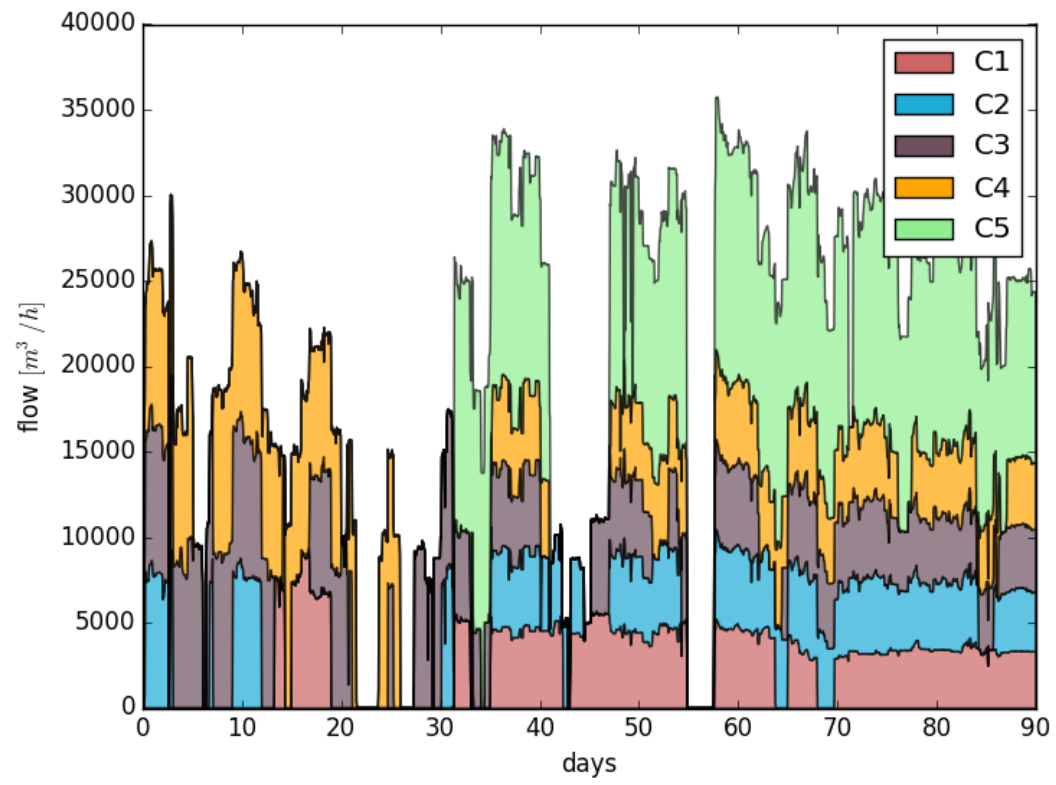

Figure 11 Flow division (the flow for each compressor $C 1-C 5$, as a function of time), for the automatic control of the compressor units (see online version for colours)

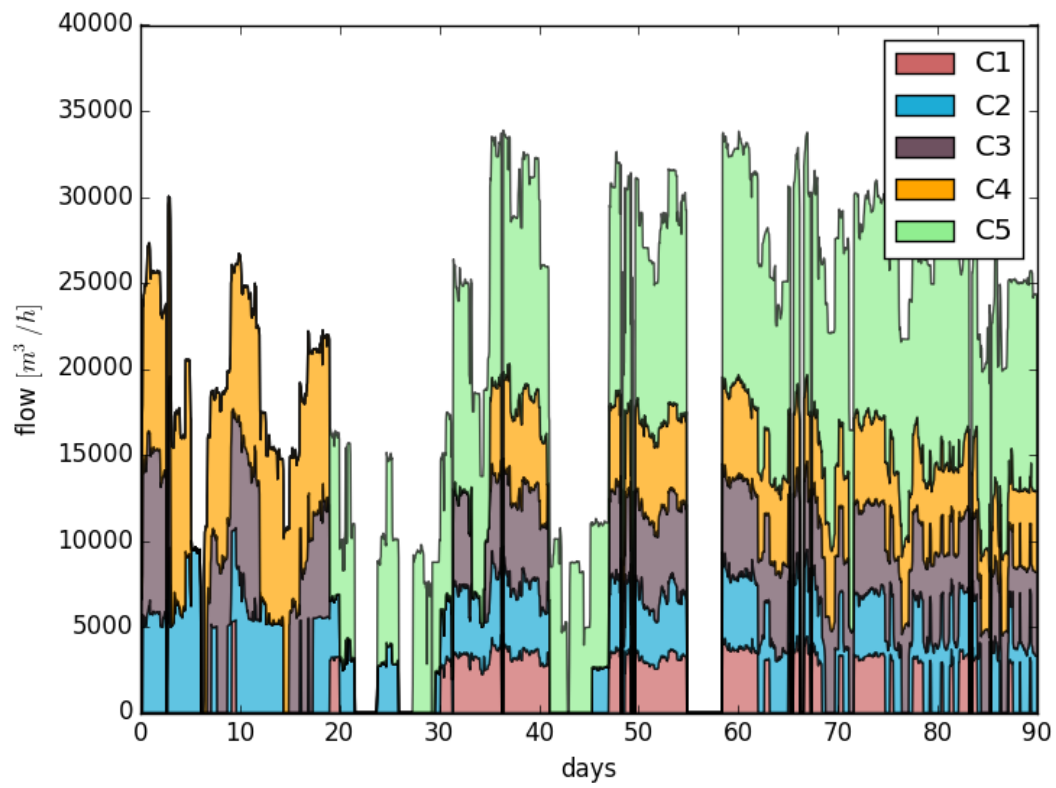


Figure 12 Comparison of estimated energy use, for the manual operation (x-axis) versus automatic operation (y-axis), for one-hour samples (see online version for colours)

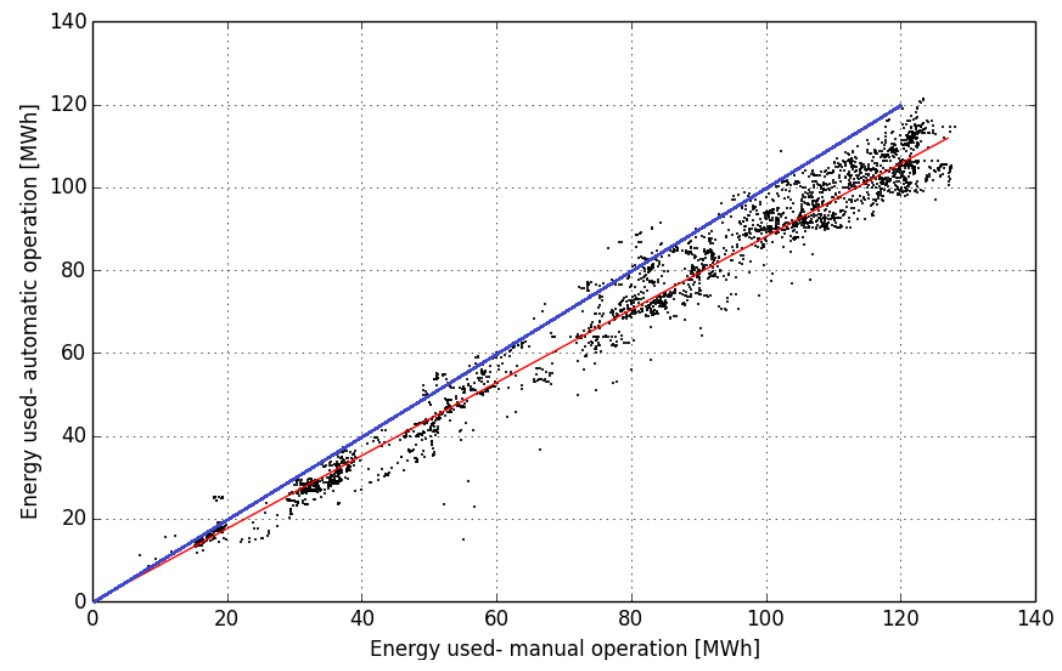

Notes: Red line - linear fit, blue line - reference line.

Figure 13 Comparison of the monthly operational costs for the manual control versus the proposed method (see online version for colours)

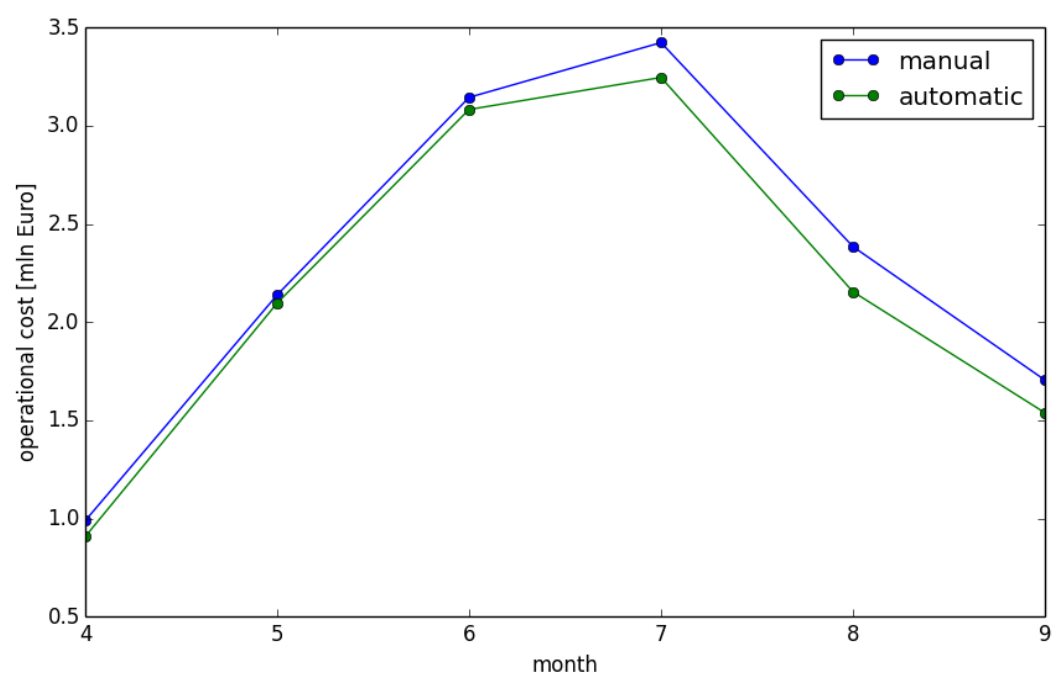

\section{Discussion and conclusions}

In this article we have presented a computational method which performs an optimal operation of compressor units in an industrial gas storage system. Our purpose is to calculate an optimal distribution of gas flow between the compressors, and the optimal discrete configuration of the compressors. We take into account that the compressor in 
the plant might be of different types with different operational parameters and power drives. We also include the possibility to operate a compressor in a 'bypass mode'. The presented optimisation algorithm takes advantages of SQP methods and dynamic programming applied to the nonlinear optimisation problem. The application of the optimisation algorithm reduces the energy consumption of the compressors and reduces the operational costs. We believe that this system is therefore a useful tool for operators of multi-compressor gas storage and transportation stations.

\section{References}

Almasi, A. (2011) 'Optimize compressor configurations for hydrocarbon applications', Hydrocarbon Processing, September, Gulf Publishing Company, Hydrocarbon Processing, (ISSN: 0018-8190).

Brent, R.P. (1973) 'An algorithm with guaranteed convergence for finding a zero of a function', in Algorithms for Minimization without Derivatives, Prentice-Hall, Englewood Cliffs, NJ, ISBN 0-13-022335-2.

Carter, R.G. (1996) 'Compressor station optimization: computational accuracy and speed', 28th Annual Meeting, Pipeline Simulation Interest Group, Paper 9605.

Cortinovis, A., Zovadell, M., Mercangoz, M., Pareschi, D., De Marco, A. and Bittant, S. (2014) 'Online adaptation of performance maps for centrifugal gas compressors', European Control Conference (ECC), Strasbourg, pp.1036-1041.

Elshiekh, T.M. (2014) 'Optimization of fuel consumption in compressor stations', Oil and Gas Facilities, Vol. 4, No. 1, pp.59-65.

Habibvand, G. and Behbahani, R.M. (2012) 'Using genetic algorithm for fuel consumption optimization of a natural gas transmission compressor station', International Journal of Computer Applications (0975-8887), Vol. 43, No. 1, pp.1-6.

Hawryluk, A., Botros, K.K., Golshan, H. and Huynh, B. (2010) 'Multi-objective optimization of natural gas compression power train with genetic algorithms', 8th International Pipeline Conference, Calgery, Alberta, Canada.

International Standard ISO 20765-1:2005(E) (2005) Natural Gas: Calculation of Thermodynamic Properties, International Organization for Standardization, Geneva, Switzerland.

Koch, T., Hiller, B., Pfetsch, M. and Schewe, L. (2015) 'Evaluating gas network capacities', MOSSIAM Series on Optimization, ISBN: 978-1-61197-368-6.

Kurz, R., Lubomirsky, M. and Brun, K. (2012) 'Gas compressor station economic optimization', International Journal of Rotating Machinery, Vol. 2012, Article ID 715017, pp.409-444.

Osiadacz, A. (1980) 'Nonlinear programming applied to the optimum control of a gas compressor station', Int J Numer Methods Eng, Vol. 15, No. 9, pp.1287-1301.

Rios-Mercadoa, R.Z. and Borraz-Sanchez, C. (2015) 'Optimization problems in natural gas transportation systems: a state-of-the-art review’, Applied Energy, Vol. 147, pp.536-555.

Rose, D., Schmidt, M., Marc, S. and Willert, B.M. (2016) 'Computational optimization of gas compressor stations: MINLP models versus continuous reformulations', Mathematical Methods of Operations Research, Vol. 83, (3), pp.409-444.

Woelfer, W. and Leuning, S. (2004) 'Well provided for with the largest natural gas storage in Western Europe', OIL GAS European Magazine, Vol. 30, No. 3, pp.116-123.

Wojdan, K., Ruszczycki, B., Michalk, D. and Swirski, K. (2014) 'Method for simulation and optimization of underground gas storage performance', Oil Gas Sci. Technol. - Rev. IFP Energies nouvelles, December, Vol. 69, No. 7.

Wong, P.J. and Larson, R.E. (1968) 'Optimization of natural-gas pipeline systems via dynamic programming', IEEE Transactions on Automatic Control, AC-13, Vol. 13, No. 5, pp.475-481. 
Xu, Y., Liu, W., Shi, C., Li, W. and He, L. (2012) 'Operation optimization method for saving compressor energy of natural gas pipeline', ICPTT 2012, November, pp.696-707.

Zhang, X. and Wu, C. (2015) 'Energy cost minimization of a compressor station by modified genetic algorithms', Engineering Letters, Vol. 23, No. 4, pp.258-268.

\section{Appendix}

\section{List of abbreviations and symbols}

$N$

$i$

$T$

$t$

$p^{s}, p^{d}$

$V_{i}(t)$

$m_{i}(t)$

$P$

$r$

$V^{N}$

$\delta h$

$\eta^{\text {mech }}(r)$

$\mathcal{P}_{V}^{d}\left(p^{s}, V^{N}, r\right)$

$\mathcal{P}_{V}^{d}\left(p^{s}, V^{N}, r\right)$

$C_{i, m_{i}(t)}^{w o r k}\left(V_{i}(t), t\right)$

$C_{\text {trans }}$

$V_{i}^{\min / \max }$

$V^{t o t}(t)$

$p_{\text {intermediate }}$ total number of compressors in the compressor unit

compressor index

the total number of simulated time-steps

the time-step in the simulation

suction pressure, discharge pressure

flow at the time-step $\mathrm{t}$ through the compressor $i$

discrete mode of the compressor $i$ at the time-step $t$

shaft power

shaft rotational speed

the normalised flow

isentropic enthalpy

efficiency function of the compressor drive

output pressure as a function of the suction pressure $p^{s}$, the rotational speed $r$, and the normalised flow $V^{N}$

output pressure as a function of the suction pressure $p^{s}$, the shaft power $P$, and the normalised flow $V^{N}$.

cost of work related to the operation of compressor $i$ at the timestep $t$ with the flow $V$

cost of switching the compressor from one configuration to another

minimal/maximal flow limit through the compressor $i$

total flow at time $t$

pressure between the compressors of stage 1 and stage 2 in a cascade configuration

$V^{\text {recalc }}\left(V, T^{\prime}, p^{\prime}, T^{\prime \prime}, p^{\prime \prime}\right) \quad$ physical flow at temperature $T^{\prime \prime}$ and pressure $p^{\prime \prime}$, recalculated from the value $V$ at temperature $T^{\prime}$ and pressure $p^{\prime \prime}$. 